\title{
Effects of Breast Motion on Lower-Body Kinematics during Running
}

\author{
Gibson Taylor M. ${ }^{1}$, Langenderfer Joseph E. ${ }^{2}$, Ustinova Ksenia I. ${ }^{1, *}$ \\ ${ }^{1}$ Doctoral Program in Physical Therapy, Central Michigan University, United States \\ ${ }^{2}$ School of Engineering and Technology, Central Michigan University, United States
}

Received December 29, 2019; Revised February 18, 2020; Accepted February 24, 2020

Copyright $@ 2020$ by authors, all rights reserved. Authors agree that this article remains permanently open access under the terms of the Creative Commons Attribution License 4.0 International License

\begin{abstract}
We measured the effects of breast motion on lower-body kinematics during overground running. Thirty female participants aged 18-60 years completed two blocks of three trials each of overground running for $25 \mathrm{~m}$ at self-selected pace under two conditions: with and without breast support provided by a sports bra. Participants' body movements were analyzed with 18 parameters characterizing breast and lower-body kinematics. Sports bra use reduced breast motion and increased about $83 \%$ of the lower-body kinematic parameters, including running velocity; stride length; foot clearance; vertical and lateral center of mass displacement; and thorax, hip, knee, and ankle range of motion (all p < 0.05). Among the changed characteristics, the stride length, center of mass displacements, thorax and knee range of motions correlated negatively ( $r=-0.25$ to -0.46$)$ with the velocity of breast motion. Therefore, the reduction of breast motion may improve lower-body kinematics during running, which may influence sport and leisure performance.
\end{abstract}

\section{Keywords Sports Bra, Sport Exercise, Gait}

\section{Introduction}

Running is a complex motion that involves whole body movement and serves multiple purposes, including a sport or leisure aerobic activity [1]. As more people engage in running to maintain healthy lifestyles and participate in sports, the investigation of factors that influence running is important.

One factor that affects running performance in females is excessive breast motion [2]. Such effects are increasing with the obesity epidemic and the availability of breast augmentation procedures [3]. Excessive breast motion during exercise increases pressure and pulling force on the
Cooper ligaments, pectoralis muscles, and ribs [4], and adds load to the thoracic and cervical spine [5]. As a result, excessive breast motion is frequently associated with discomfort, embarrassment, and postural abnormalities [6-7].

Proper breast support has been proposed to reduce discomfort and improve performance in sports and other activities requiring intensive movement of the whole body. The first sports bra was developed in 1977 to provide additional breast support that regular bras could not offer [8]. Since then, the sports bra industry has advanced, with the design of increasingly complex and competitive products [6]. This industry development has, in turn, stimulated research on the effects of various forms of breast support on women's comfort during exercise. Wearing a sports bra during physical activity has been accepted to decrease pain and discomfort [6] by minimizing vertical and lateral breast displacement [9]. It has been also agreed that effectiveness of sports bras in reducing undesired breast motion varies depending on the product type and/or brand and the type of physical activity [10-12].

To date, the effects of breast motion reduction have been studied mostly during running, the most typical high-intensity physical activity. Changes in breast kinematics are well documented, but less is known about changes in the kinematics of running related to excessive breast motion. A few studies have been performed, but they have produced limited and ambiguous results. McGhee et al. [13] reported that different breast support conditions influenced stride frequency among women running on a treadmill and in water. The researchers interpreted this effect as a protective mechanism used by the participants to lessen breast discomfort. In contrast, White et al. [9] found that stride length and frequency during running did not differ between breast support conditions. This research group also showed, that the running body produces higher medial impact forces under the no-bra condition [14]. 
Finally, Milligan et al. [15] found that high breast support reduced the torso and upper arm ranges of motion (ROMs) in female runners, approximating a running pattern to that is more economical and beneficial for long distances. Thus, some evidence of changes in running patterns due to the wearing of sport bras has been accumulated, but it is insufficient to fully understand the effects of breast motion on the kinematics of self-paced running.

Considering these shortcomings, the present study was designed to analyze and compare lower-body kinematics during overground running with and without breast support. We hypothesized that unsupported breast motions would influence running kinematics in the lower extremities and trunk. The results could contribute to women's improved performance in sports and physical activities.

\section{Materials and Methods}

\subsection{Subjects}

With institutional review board approval, 30 female participants aged 18-60 volunteered and gave written informed consent to participate in this study. Due to data corruption, results from two participants were not included in the analysis. All participants were currently aerobically active on at least 1 day a week, had no history of breast surgery, and had not given birth or breast fed within the last year. Participants' breast size, measured as the difference between breast circumference and rib circumference, ranged widely from 8.5 to $21.5 \mathrm{~cm}$, corresponding to the commonly accepted bra cup sizes of AA-DD.

The initial sample size of 30 subjects was determined based on the literature review, and consistent with sample size used in most studies testing medical devices and apparel. Interim power analysis was performed after collecting data from the first 10 participants and the results ensured power of at least $85 \%$ at significance level of 0.05 with planned number of subjects.

\subsection{Experimental Procedure and Data Analysis}

Participants completed two blocks of three trials each of overground running for $25 \mathrm{~m}$ at a self-selected speed under two randomized experimental conditions: with supported (sports bra) and unsupported (control) breasts. The Shefit Ultimate Sports Bra (Shefit, Hudsonville, MI, USA) was selected as the experimental sports bra from among five products based on its quality, as tested and described in a previous work [12]. A trained bra fitter ensured that the sports bra fit each participant properly. Before the trials were performed, the participants were instructed to practice overground running to familiarize themselves with the distance and movement pattern. Overground running was chosen as an experimental paradigm to increase applicability to normal physical activity patterns, with no limitation to treadmill running.

During running, participants' body movements were captured with a Vicon T160 system (Vicon Industries, Inc, Centennial, CO, USA) at $100 \mathrm{~Hz}$. Subjects wore 47 retro-reflective 1 -cm markers placed strategically over the following landmarks: the bilateral acromioclavicular joint line, mid-arm, lateral epicondyle, radial styloid, dorsal mid-carpals, nipple, anterior superior iliac spine, posterior superior iliac spine, greater trochanter, lateral malleolus, mid-leg, calcaneus, and lateral knee joint line; the eighth thoracic vertebra, seventh cervical vertebra, suprasternal notch, xiphoid process, right mid-back, occiput and frontalis line of the head; and four markers on each breast in the area surrounding the nipple (Figure 1). Previous studies have determined that the center of the nipple is a reliable landmark for the placement of a retro-reflective marker to measure vertical, mediolateral, and anteroposterior breast displacement in relation to the movement of the trunk [16]. The markers were placed directly over the nipple for control trials, and indirectly on the nipple over the bra fabric for the sports bra trials.

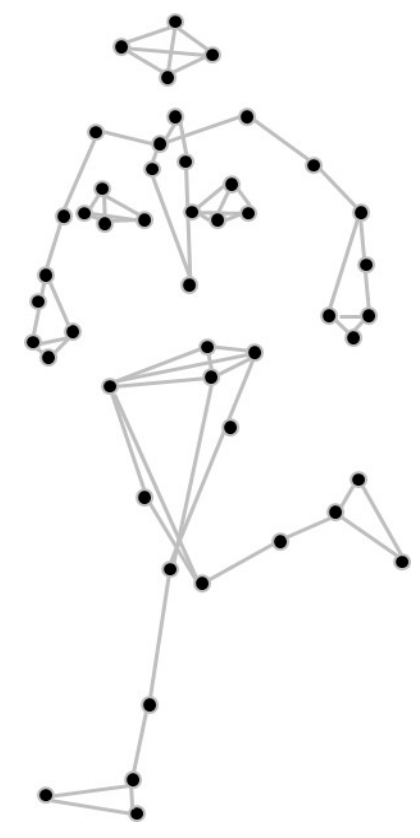

Figure 1. Standard marker placement setup with 47 retro-reflective markers on anatomical landmarks and the bilateral breast

The Vicon Plug-In Gait model, adjusted to accommodate the breast markers, was used to calculate two groups of outcome measure: breast kinematics and lower-body kinematics (spatiotemporal characteristics, body stability, and angular joint displacement).

Breast kinematics were computed using trajectories of nipple marker displacement from the right breast relative to thorax marker displacement in three planes and included the ranges of lateral and vertical breast displacement and maximal lateral and vertical breast velocities.

Lower-body kinematics were represented by the spatiotemporal characteristics of running and were 
calculated using trajectories of the right lower extremity, including cadence (number of steps per minute), velocity (distance of the body over step time), single support time (relative time of one foot's contact with the ground), stride length (distance between two consecutive heel strikes of the same foot), step width (maximum perpendicular distance between heels), and foot clearance (maximum distance from the toes to the ground). Body stability measures included the center of mass (COM) average lateral displacement (root mean square of the COM per step in the lateral direction), COM lateral range (difference between maximum and minimum lateral COM displacements), COM average vertical displacement (root mean square of the COM per step in the vertical direction), and COM vertical range (difference between maximum and minimum COM displacements in the vertical direction). Angular joint displacement measures included the rotational thorax ROM in the horizontal plane and the hip, knee, and ankle ROMs in the sagittal plane. All measures except the thorax ROM were calculated for the right lower extremity per step and averaged across at least five to six steps.

Statistically, differences in running and breast kinematics between the supported and unsupported conditions were assessed using parametric dependent $t$ tests with a significance level of $p<0.05$, after data normality distribution was confirmed with the Kolmogorov-Smirnov test. Pearson coefficients were used to examine correlations between the velocity of breast motion and measures of lower-body kinematics that differed significantly between conditions. Coefficients of $r>0.25$ indicated mild correlation, and those of $r>0.40$ indicated moderate correlation [17].

\section{Results}

Sports bra use resulted in significant reductions in all breast kinematic parameters, ranging from $44 \%$ to $61 \%$ for lateral and vertical displacement, and from $50 \%$ to $52 \%$ for vertical and lateral peak velocities (all $p<0.05$; Table 1 ). Among spatiotemporal characteristics of running, cadence $(p=0.051)$, velocity $(p=0.008)$, single support time $(p=$ $0.011)$, stride length $(p=0.003)$, and foot clearance $(p<$ 0.001) were increased significantly under the breast-supported condition (Table 2). Step width and cadence did not show significant difference but tendency to change. Sports bra use significantly increased the average $(p=0.007)$ of vertical $(p=0.007)$ and lateral $(p=0.026)$ COM displacement. The range of COM displacement was also increased in vertical direction only $(p=0.005)$. No significant difference found for lateral COM range ( $p=$ 0.222) (Table 2). ROM increased during running with supported breasts for all angular displacements $(p<0.05)$.

Across both breast support conditions, mild to moderate negative correlations were found between breast velocities and stride length ( $r=-0.46$ and $r=-0.37$ ). The velocities of breast motion also correlated with the COM vertical displacement range $(r=-0.44)$ and average $(r=-0.26$ and $r$ $=-0.34)$, and with the thorax and knee ROMs $(r=-0.28$ and $r=-0.25$, respectively; Table 3). No correlation was found between breast motion and other lower-body kinematic measures, including velocity, foot clearance, and hip ROM.

Table 1. Breast kinematics measures under two overground running conditions ( $\mathrm{n}=30)$.

\begin{tabular}{|c|c|c|c|}
\hline \multirow{2}{*}{ Breast kinematics measures } & \multicolumn{2}{|c|}{ Running conditions } & \multirow{2}{*}{ P-value } \\
\cline { 2 - 4 } & Control & Sports Bra & $<0.001^{*}$ \\
\hline Range Lateral Displacement (cm) & $6.68 \pm 3.09$ & $1.7 \pm 0.48$ & $<0.001^{*}$ \\
\hline Range Vertical Displacement (cm) & $4.43 \pm 2.18$ & $36.6 \pm 17.0$ & $<0.001^{*}$ \\
\hline Peak Lateral Velocity (cm/s) & $74.0 \pm 34.1$ & $23.1 \pm 14.9$ & $<0.001^{*}$ \\
\hline
\end{tabular}

Values are presented as means \pm standard deviations. ${ }^{*} \mathrm{p}<0.05$ 
Table 2. Lower-body kinematics measures under two overground running conditions (n=30)

\begin{tabular}{|c|c|c|c|}
\hline & \multicolumn{2}{|c|}{ Running conditions } & \multirow{2}{*}{ P-value } \\
\hline & Control & Sports Bra & \\
\hline \multicolumn{4}{|c|}{ Spatiotemporal measures } \\
\hline Cadence (steps/min) & $179 \pm 21.4$ & $183 \pm 19.8$ & 0.051 \\
\hline Velocity (m/s) & $3.49 \pm 0.88$ & $3.71 \pm 0.72$ & $0.008^{*}$ \\
\hline Single Support Time (\%) & $40.6 \pm 6.04$ & $38.8 \pm 4.74$ & $0.011^{*}$ \\
\hline Stride Length (m) & $2.31 \pm 0.33$ & $2.42 \pm 0.31$ & $0.003^{*}$ \\
\hline Step Width (cm) & $6.21 \pm 2.30$ & $6.75 \pm .2 .53$ & 0.060 \\
\hline Foot Clearance $(\mathrm{cm})$ & $25.4 \pm 9.77$ & $28.9 \pm 8.70$ & $<0.001^{*}$ \\
\hline \multicolumn{4}{|c|}{ Body stability measures } \\
\hline COM Average Lateral Displacement (mm) & $1.14 \pm 0.59$ & $1.24 \pm 0.65$ & $0.026^{*}$ \\
\hline COM Lateral Range (mm) & $119 \pm 76.1$ & $124 \pm 83.4$ & 0.222 \\
\hline COM Average Vertical Displacement (mm) & $4.38 \pm 0.75$ & $4.68 \pm 0.64$ & $0.007^{*}$ \\
\hline COM Vertical Range (mm) & $97.8 \pm 22.7$ & $105 \pm 19.5$ & $0.005^{*}$ \\
\hline \multicolumn{4}{|c|}{ Angular joint measures } \\
\hline Thorax $\mathrm{ROM}^{\circ}$ & $32.0 \pm 8.63$ & $34.9 \pm 6.51$ & $0.001^{*}$ \\
\hline Hip ROM ${ }^{\circ}$ & $76.3 \pm 14.4$ & $80.2 \pm 18.0$ & $0.007^{*}$ \\
\hline Knee ROM $^{\circ}$ & $101 \pm 16.6$ & $109 \pm 17.0$ & $0.004 *$ \\
\hline Ankle $\mathrm{ROM}^{\circ}$ & $55.0 \pm 7.16$ & $56.8 \pm 7.61$ & $0.019 *$ \\
\hline
\end{tabular}

Values are presented as means \pm standard deviations. ${ }^{*} \mathrm{p}<0.05$. COM -Center of Mass; ROM - Range of Motion

Table 3. Pearson coefficients of correlation between breast and lower-body kinematics measures $(n=30)$.

\begin{tabular}{|c|c|c|}
\hline \multirow{2}{*}{ Lower-body kinematics measures } & \multicolumn{2}{|c|}{ Breast Kinematics Measures } \\
\hline & Maximal Velocity Lateral (cm/s) & Maximal Velocity Vertical (cm/s) \\
\hline Velocity $(\mathrm{m} / \mathrm{s})$ & $r=-0.11$ & $r=-0.10$ \\
\hline Stride Length (m) & $r=-0.46$ & $r=-0.37$ \\
\hline Foot Clearance $(\mathrm{cm})$ & $r=-0.14$ & $r=-0.08$ \\
\hline COM RMS Vertical Displacement (mm) & $r=-0.26$ & $r=-0.34$ \\
\hline COM Vertical Range (mm) & $r=-0.44$ & $r=-0.18$ \\
\hline Thorax Horizontal $\mathrm{ROM}^{\circ}$ & $r=-0.28$ & $r=-0.21$ \\
\hline Hip Sagittal ROM ${ }^{\circ}$ & $r=0.04$ & $r=0.08$ \\
\hline Knee Sagittal ROM ${ }^{\circ}$ & $r=-0.19$ & $r=-0.25$ \\
\hline
\end{tabular}

COM -Center of Mass; ROM - Range of Motion; RMS- Root Mean Square 


\section{Discussion}

The present study investigated the effects of breast support on lower-body kinematics during overground running. The results indicated that about $85 \%$ of kinematic parameters examined were affected by unsupported breast motion. This finding was partially supported by the mild to moderate correlations observed between the changed kinematic parameters and breast motion velocities. The findings suggest that sports bra use has the potential to significantly improve performance in overground running.

Running is a complex activity that involves whole body movement, and is traditionally assessed by examination of multiple parameters. Thus, several questions arise when considering the significance of the present findings. The first question is whether changes in lower-body kinematics indicate improvement in running performance. Traditionally, the quality of running is defined by velocity, with faster considered to be better. Similar to the gait pattern, the velocity of running is a derivative of multiple factors, such as the step or stride length and frequency. Increased velocity is achieved initially through increasing step length, followed by increasing cadence [18]. In turn, the increase of step or stride length requires mechanically greater ROMs in the major participating joints of the lower extremities and trunk [19]. Thus, the increased spatiotemporal and angular joint parameters during sports bra use could be considered to reflect improvements or to be positive effects. The results of our study are consistent with those of another study showing that proper breast support reduces discomfort, thereby improving running performance [9]. However, the authors also reported no effect of breast support on stride length or frequency during overground running. These discrepancies in results may arise from the use of different experimental designs. In our study, participants ran a longer distance, which allowed them to achieve faster speeds that might have amplified the effect of unsupported breast motion on running performance. Another explanation different results may arise from the self-selected speed of running that was not controlled in our study.

Another controversy derives from the study of Milligan et al. [15], who reported reduced torso and upper arm ROM when a bra providing high breast support was worn. During treadmill running, this bra also reduced the breast ROM compared with the use of a bra providing low (and less effective, according to the authors) breast support. In our study, proper breast support increased trunk rotation. This effect could be explained by participants' application of a strategy to minimize unpleasant breast oscillation when running without a bra by "squeezing" the breasts between their upper arms. This strategy likely did not mechanically restrict undesired breast motion but may have helped to create a subjective feeling of reduced motion-related discomfort. As a side effect, this self-restricting strategy could reduce trunk ROM compared with that observed when running with a sports bra. This statement is rather speculative, as changes in upper extremity motion were not analyzed in the study.

Another effect observed in this study was increased COM displacement when running with breast support. This finding is commonly interpreted as reflecting reduced stability, and thus unlikely to indicate improvement in running performance. Although this interpretation holds for the majority of postural control studies, we argue that it was an effect of increased running speed, rather than a sign of instability, in the present study.

The final important question is whether the mild negative correlation between breast and lower-body motion constitutes evidence of the effects of sports bra use on the kinematics of running. Statistically, coefficients < 0.4 do not indicate strong correlations unless the experimental condition is unfavorable and affected by factors that cannot be excluded from analysis. An example of such a factor would be the arrangement of all segments of the running body in kinematic chains, in which motions of all segments are mutually dependent. These natural mechanics of the human body dictate that increased ROM in the lower extremities and trunk generates excessive motion of the breast, which is obviously attached to the trunk. However, that was not the case in our study, as correlations between lower-body and breast motions were negative. Thus, the mild correlations, observed against all biomechanical properties of a moving body, could signify increased significance of the effect of breast support on overground running performance.

Future studies may investigate such correlation effects during longer or faster overground running, as velocity reduction, with corresponding changes in lower-body kinematics, may have been a protective mechanism to reduce discomfort during overground running with unsupported breasts in this study. This statement is rather speculative and based on the results of other works [13, 20], as the present study did not address the question of discomfort due to lack of breast support while running.

\section{Conclusions and Limitations}

In summary, this study showed that sports bra use alters lower-body kinematics, thereby improving overground running. Further investigation of this topic would provide a better understanding of how to enhance sports bra design, which may lead to improved sports performance, as the present study has several limitations. First, the sample of female participants was relatively homogenous, with small to medium-sized breasts; few participants had large breasts (D cup and larger). Second, the convenience sample may not be representative of the physically active female population. Third, the running trials were performed over a short distance, which may have influenced certain kinematic parameters. All of these limitations will be 
addressed in future works.

\section{Acknowledgements}

This study was funded by Shefit, Inc, Hudsonville, MI, USA. The authors wish to thank Amy Bunting for bra fitting.

\section{REFERENCES}

[1] S. Dugan, K, Bhat. Biomechanics and analysis of running gait. Phys Med Rehabil Clin N Am, 16, 603-621, 2005.

[2] J. Scurr, J. White, W. Hedger. The effect of breast support on the kinematics of the breast during the running gait cycle. J Sports Sci, 28, 1103-1109, 2010.

[3] N. Brown, J. Scurr. "Breasts are getting bigger". Where is the evidence? J Anthropol Sci, 20, 94, 237-44, 2016.

[4] A. Gefen, B. Dilmoney. Mechanics of the normal women's breast. Technol Health Care, 15, 259-271, 2007.

[5] E. L. Wood, J. White, A. Milligan, B. Ayres, W. Hedger, J. Scurr. Predictors of Three-Dimensional Breast Kinematics during Bare-Breasted Running. Med Sci Sports Exerc, 44, 1351-1357, 2012.

[6] K. A. Bowels, J. Steele, B. Munro. Features of sports bras that deter their use by Australian women. J Sci Med Sport, 15, 195-200, 2012.

[7] N. Brown, J. White, J. Scurr. The experience of breast pain (mastalgia) in female runners of the 2012 London Marathon and its effect on exercise behavior. Br J Sports Med, 48, 320-325, 2014.

[8] K. Schuster. Equipment update: jogging bras hit the streets. Phys Sportsmed, 4, 125 - 128, 1979.

[9] J. White, J. Scurr, W. Hedger. A comparison of three-dimensional breast displacement and breast comfort during overground and treadmill running. $\mathrm{J}$ of Appl Biomech, 27, 47-53, 2011.

[10] D. Lorentzen, L. Lawson. Selected Sports Bras: A Biomechanical analysis of breast motion while jogging. The Physician and Sports Medicine, 5, 128-139, 1987.

[11] M. S. A. Hadi. Sports brassiere: is it a solution for mastalgia. Breast J, 6, 407-409, 2000.

[12] T.M. Gibson, N. Balendra, K.I. Ustinova, J.E. Langenderfer. Reductions in Kinematics from Brassieres with Varying Breast Support. Int J Exerc Sci, 12, 402-411, 2019.

[13] D. E. McGhee, B.M. Power, J.R. Steele. Does deep water running reduce exercise-induced breast discomfort. $\mathrm{Br} \mathrm{J}$ Sports Med, 41, 879-883, 2007.

[14] White JL, Scurr JC, Smith NA. The effect of breast support on kinetics during overground running performance. Ergonimics, 52, 492-498, 2009

[15] A. Milligan, C. Mills, J. Corbett, J. Scurr. The influence of breast support on torso, pelvis and arm kinematics during a five kilometer treadmill run. Hum Mov Sci, 42, 246-260, 2015.

[16] B. Mason, K. Page, K. Fallon. An analysis of movement and discomfort of female breast during exercise and the effects of breast support in three cases. J Sci Med Sport, 2, 134-144, 1999.

[17] S. Landau, B. S. Everitt. A handbook of statistical analyses using SPSS. Chapman \& Hall/CRC Press LLC, 2004.

[18] S. Õunpuu. The biomechanics of walking and running. Clin Sports Med, 13, 843-863, 1994.

[19] M.S. Orendurff, T. Kobayashi, K. Tulchin-Francis, A. M. H. Tullock, C. Villarosa, C. Chan, E. Kraus, S. Strike. A little bit faster: Lower extremity joint kinematics and kinetics as recreational runners achieve faster speeds. J Biomech, 71, 167-175, 2018

[20] N. Shivitz. Adaptation of vertical ground reaction force due to changes in breast support in running. Corvallis, Oregon: Oregon State University 2002. 